\title{
Genomic insights into tuberculosis
}

\author{
James E. Galagan
}

Abstract | Prevalent since pre-history, human tuberculosis - caused by the pathogen Mycobacterium tuberculosis - remains a major source of death worldwide. Moreover, increasing drug resistance poses the threat of disease resurgence. However, the expanding application of genomic techniques is providing new avenues for combating this old foe. Whole-genome sequencing, comparative genomics and systems biology are generating new insights into the origins and ongoing evolution of M. tuberculosis, as well as the molecular basis for its pathogenicity. These have important implications for our perspective of the disease, development of new drugs and vaccines, and treatment of patients using existing therapeutics.

\section{Active disease \\ A Mycobacterium tuberculosis infection associated with clinical symptoms of tuberculosis. \\ Granuloma \\ An organized nodule of inflammatory cells that is associated with a range of diseases, including tuberculosis. A tuberculosis granuloma consists of a core of infected macrophages surrounded by phagocytes that are enclosed in a mantle of lymphocytes and a fibrous cuff.}

Professional pathogen An organism adapted for a pathogenic lifestyle.

Department of Biomedical Engineering, Bioinformatics program and National Emerging Infectious Diseases Laboratory, Boston University, 34 Cummington Mall 1002, Boston Massachusetts 02215; and Broad Institute of Massachusetts Institute of Technology and Harvard, 7 Cambridge Center, Cambridge, Massachusetts 02142, USA e-mail:jgalag@bu.edu doi: 10.1038/nrg3664 Published online 25 March 2014
Every minute, another three people in the world die of tuberculosis. With more than 8 million new cases of active disease and nearly 1.5 million deaths annually, tuberculosis is a global health emergency of overwhelming proportions ${ }^{1}$. The causal pathogen - the bacterium Mycobacterium tuberculosis - is transmitted by inhalation. In the lungs, $M$. tuberculosis is phagocytosed by macrophages, which are thought to be the predominant host cell for the majority of the infectious life cycle. Internalization by macrophages triggers an immune response, the recruitment of additional monocytes and, ultimately, the formation of a granuloma that effectively contains the infected cells. The success of this microorganism is partly due to its ability to survive within macrophages in a granuloma for months and even decades in an asymptomatic state ${ }^{2-4}$. It has been estimated that one-third of the human population may harbour M. tuberculosis in this state ${ }^{5}$. For transmission to occur this containment must fail (which can be due to changes in the host immune status) or be overcome. When the granuloma breaks down, infectious bacilli can be released into the airways to be expelled and transmitted to the next host. Although this outline of the life cycle is generally accepted ${ }^{2,3}$, many details of this cycle and the mechanisms that allow $M$. tuberculosis to survive in the host remain poorly understood.

M. tuberculosis is one member of a group of organisms known as the M. tuberculosis complex (MTBC) that also includes numerous strains of $M$. tuberculosis, the human pathogen Mycobacterium africanum and a clade of animal-infecting mycobacteria (including Mycobacterium bovis). Some non-tuberculosis mycobacteria are capable of causing infections in humans or animals, but the majority are common environmental organisms that typically live in soil ${ }^{6}$. The mycobacteria, in turn, are members of Gram-positive Actinomycetes, which include numerous common soil and aquatic bacteria. The story of M. tuberculosis is therefore a story of how what was most likely a soil bacterium evolved to become one of the most successful human pathogens in history.

In this Review, I describe how the application of genomics to the study of tuberculosis is accelerating our understanding of this ancient disease. Through the expanding use of genome sequencing and comparative genomic analyses, the research field has gained new insights into the origins of $M$. tuberculosis. This in turn has provided knowledge into how M. tuberculosis evolved to become a 'professional pathogen' of humans and how it continues to evolve to evade our clinical efforts through drug resistance. The extension of sequencing technology to systems biology has further yielded insights into the adaptations that enable M. tuberculosis to survive in the host. Together with an extensive literature on cellular biology and immunology of tuberculosis, these data give rise to an emerging picture of an organism that has evolved over an extended period of time to adapt to, and possibly orchestrate changes in, the host immune system.

\section{The emergence of a human pathogen}

Tuberculosis has affected humans for thousands of years. The ancient association of tuberculosis with humanity is supported by literary descriptions, by morphological evidence in human fossils and by the identification of mycobacterial DNA in human remains using genotyping methods, including PCR of the IS6110 repeat sequence, spoligotyping or PCR amplification, and sequencing of the D1 deletion region (BOXES 1,2; see Supplementary 
information S1 (table)). The initial analyses of these data led to the hypothesis that human-infecting M. tuberculosis arose through a zoonotic transmission of an ancient $M$. bovis strain from cows during domestica$\operatorname{tion}^{7,8}$ (BOX 1), which is similar to the zoonotic origins of other human pathogens such as measles and influenza viruses. However, it was the subsequent application of genomics that forced a re-evaluation of the origins of M. tuberculosis in humans.

Fresh phylogenetic insights from genome sequencing. Insights into the emergence of $M$. tuberculosis as a human pathogen have come from several genomic studies, such as whole-genome sequencing (WGS) of M. tuberculosis, the characterization of the genetic diversity of modern M. tuberculosis strains and comparative genomic analyses with related species (including other members of the MTBC and smooth tubercle bacilli (STBs)).

The WGS of the first strain of M. tuberculosis - the widely used laboratory strain $\mathrm{H} 37 \mathrm{Rv}$ - was a landmark in the study of this disease 9 . A little more than 100 years after the bacterium was isolated by Robert Koch, it was revealed to have a GC-rich genome of $4.4 \mathrm{Mb}$ that contains $\sim 4,000$ genes. The genome sequence also enabled the development of DNA microarrays that could be used to probe the gene content of closely related mycobacteria through comparative genome hybridization $^{10}$. Such studies, complemented by pulse-field gel electrophoresis approaches ${ }^{11,12}$, resulted in the identification of variable genomic regions that are present in some but not all MTBC strains. The distribution of these regions led to the construction of a phylogenetic

\section{IS6110 repeat sequence An insertion element specific to the Mycobacterium tuberculosis complex. \\ Spoligotyping \\ A PCR-based method for genotyping Mycobacterium tuberculosis strains on the basis of the presence or absence of clustered regularly interspaced short palindromic repeat (CRISPR) spacer sequences. \\ D1 deletion \\ A genomic region \\ deleted specifically in Mycobacterium tuberculosis relative to the M. tuberculosis complex.}

Zoonotic transmission The transmission of an animal disease to humans.

Smooth tubercle bacilli (STBs). Mycobacteria that show a smooth colony morphology on culture media.

\section{Pulse-field gel} electrophoresis A technique for separating large DNA molecules in a gel matrix using a modulating electric field.

Pre-Columbian Pertaining to the time period before the arrival of Christopher Columbus in the Americas.

\section{Box 1 | Tuberculosis in the ancient world}

Few diseases have had an association with human beings that is as long and impactful as that of tuberculosis. Literary descriptions that are suggestive of tuberculosis date back millennia and are found in Chinese medical texts ${ }^{140}$ $(\sim 2,700 \mathrm{BC})$, the Vedic writings of India ${ }^{141}(\sim 1,500 \mathrm{BC})$, the Old Testament of the Bible ${ }^{142}(\sim 1,300-400 \mathrm{BC})$ and the writings of Hippocrates ${ }^{143}(460 \mathrm{BC})$. These and other texts refer to a disease that is variously called schachepheth (Hebrew), sosha (Sanskrit), yaksmā or xoy (Hindi) and phthisis (Greek). Phthisis translates as 'wasting away', which describes the symptomatic course of pulmonary tuberculosis that also gave rise to its common nineteenth century name consumption. The pathophysiological association of tuberculosis with the development of a granuloma, or tubercle, gave the disease its modern name.

The ancient association of tuberculosis with humanity has been supported by archaeological data and, more recently, by data obtained using molecular techniques. Although tuberculosis infections are most common in the lungs, they can occur in essentially any body part. Infection of the spine - termed Pott's disease - results in the destruction of intervertebral disks and vertebral bodies which, in the most severe cases, leads to spinal collapse and hunchback. Such characteristic anatomical changes provide a nonspecific signature of possible tuberculosis infection that is recognizable in fossil remains ${ }^{144}$. This can be supported by the identification of mycobacterial DNA through genotyping methods, including PCR confirmation of the IS6110 repeat sequence, spoligotyping or PCR amplification and sequencing of the D1 deletion region. However, each of these methods has limitations in its ability to resolve closely related lineages ${ }^{145}$. More recently, whole-genome sequencing has been applied to resolve strain phylogeny with high resolution ${ }^{146}$. In all cases, extreme care is required in both the applications of such methods and the interpretation of the results owing to the possibility of contamination with modern mycobacteria ${ }^{144}$. Nonetheless, compelling data have been generated, and many questions have been raised (see Supplementary information S1 (table)).

Archaeological investigations of tuberculosis have been most extensively applied to ancient Egyptian mummies. The first and most famous case ${ }^{147}$ was based on morphological changes in the mummified remains of Nesparehan, who was a high priest of Amon under Ramses II (1,304-1,237 BC). Subsequent studies using IS6110 typing confirmed the presence of Mycobacterium tuberculosis complex (MTBC) throughout Egyptian history back to the pre-dynastic period $(\sim 3,400 \mathrm{BC})^{145,148-151}$. It has been estimated that up to $25 \%$ of Egyptians who were later mummified may have suffered from tuberculosis ${ }^{152}$. Spoligotyping supported the presence of $M$. tuberculosis and Mycobacterium africanum, but not Mycobacterium bovis (although M. bovis was probably in existence). Other reports confirm that tuberculosis was geographically widespread with fossil evidence from fourth millennium BC Germany ${ }^{153}$, third millennium BC Italy ${ }^{154}$, Sweden, Denmark ${ }^{155}$ and Rome, and possibly also first millennium China ${ }^{156}$. The oldest fossil evidence so far of human tuberculosis comes from the 9,000-year-old remains of a woman and an infant that were recovered from a submerged site in the Eastern Mediterranean ${ }^{157}$. Morphological evidence, the mycobacterial lipids and D1 deletion region sequencing indicate not only species of the MTBC but also specifically human-infecting M. tuberculosis, which suggests that the modern version of tuberculosis already existed during the Neolithic period.

The evidence that human tuberculosis is coincident with animal domestication gave rise to the hypothesis that human-infecting M. tuberculosis arose through a zoonotic transmission of an ancient M. bovis strain from cows ${ }^{7,8}$, which is similar to the zoonotic origins of other human pathogens such as measles and influenza. However, some aspects of the archaeological record conflict with this scenario. The identification of molecular evidence of MTBC in pre-Columbian human remains from Peru ${ }^{158,159}$ and Chile ${ }^{160}$ suggests that some members of the MTBC may have crossed to the new world via the Bering Strait during the Late Pleistocene before widespread animal domestication. Moreover, typical lesions of tuberculosis have been identified in North American bovids ${ }^{161}\left(125,000-8,000\right.$ years ago) and mastodons ${ }^{162}$ (38,000-10,000 years ago), and in a Bison fossil from a natural trap cave in Wyoming, USA, which revealed molecular evidence that mycobacterial DNA is more closely aligned to modern M. tuberculosis than to M. africanum and M. bovis. These archaeological data suggest that, even if tuberculosis arose as a zoonosis, the emergence of tuberculosis is likely to predate animal domestication. 


\section{Box 2 | Genomic methods for molecular epidemiology}

Genomics is enhancing molecular epidemiological methods as applied to tuberculosis. They have been used to address numerous questions, the answers to which are advancing clinical practice ${ }^{163}$. These methods have also been applied to the bioarchaeology of tuberculosis (BOX 1). Such investigations typically rely on the ability to genotype clinical isolates, and the methods for doing so are varied and increasing.

\section{Spoligotyping}

Spoligotyping uses PCR and reverse-hybridization blotting to assess the genetic diversity of a locus that consists of 36 -bp direct repeats, which alternate with unique spacers that are $35-41$ bp in length ${ }^{30,164,165}$. Called the direct repeat (DR) locus, it is now recognized that this is an instance of a clustered regularly interspaced short palindromic repeat (CRISPR) locus found in other bacteria. In other bacteria, these loci are associated with the CRISPR-Cas (CRISPRassociated protein) system that functions as a form of bacterial acquired immunity against exogenous DNA elements ${ }^{16,167}$. Spoligotyping generates a 'fingerprint' that indicates the presence or absence of individual spacer sequences. It can resolve species identity to a coarse resolution that is sufficient, in some cases, to differentiate Mycobacterium tuberculosis from Mycobacterium bovis and Mycobacterium africanum. However, it cannot resolve M. tuberculosis complex (MTBC) strains ${ }^{168}$. Despite this limitation, spoligotyping is fast and inexpensive, and a large database of spoligotyped strains is publically available ${ }^{30}$.

\section{Restriction fragment length polymorphism with IS6110}

Restriction fragment length polymorphism (RFLP) with IS6110 uses restriction digestion of genomic DNA followed by blotting and labelling with a probe that contains the IS6110 mobile element ${ }^{168}$. Differences in both the number and the location of insertion sites for IS6110 elements result in different probe banding patterns. RFLP with IS6110 has a higher resolution than spoligotyping and can distinguish between MTBC strains. However, it is time-consuming owing to the need for substantial amounts of DNA ${ }^{168}$. IS6110 is specific to the MTBC ${ }^{169,170}$, and thus PCR confirmation of its presence can also be used to confirm MTBC (BOX 1). RFLP has also been used with probes for different repeat elements.

\section{Variable-number tandem-repeat typing}

Variable-number tandem-repeat (VNTR) typing characterizes the number of tandem repeats in 24 different loci by assessing the length of amplicons that are derived from PCR with primers specific to each locus ${ }^{171}$. The resolution and accuracy of this method is still being studied. Its advantage is the ease of implementation.

\section{Multispacer sequence typing}

Multispacer sequence typing (MST) uses a sequence-based analysis of selected intergenic regions that are present in a range of strains, but the length or sequence of which varies between strains ${ }^{172}$.

\section{Whole-genome sequencing}

Whole-genome sequencing (WGS) uses deep sequencing to sample and, in some cases, assemble the genomes of clinical isolates. In principle, WGS can simultaneously provide complete epidemiological, drug resistance and even potentially functional information. In practice, there are currently many limitations and trade-offs. The current state of sequencing technology imposes constraints. The fastest and most cost-effective method is shotgun sequencing to generate short reads that are aligned to a reference genome. Illumina instruments are currently the most widely used, although lon Torrent may also be used. As only short reads ( $40-250 \mathrm{bp}$ ) are generated, this approach does not typically capture long repeat regions. In M. tuberculosis, in particular, insertion elements and PE-PPE genes (which constitute a highly repetitive class of antigenic genes) are often excluded. Short reads are also likely to under-sample genome rearrangements. Paired-end sequencing and jumping libraries can help to mitigate these issues. Advances in genome assembly from short reads can also generate longer contiguous sequences ${ }^{173-177}$. However, the comparison to a reference sequence makes it difficult to detect regions that are specific to the sequenced strain, and the use of different references can complicate analyses. In addition, Illumina instruments show a GC bias such that GC-rich and AT-rich regions are under-sampled (although new PCR-free methods partially ameliorate this). This effect is exacerbated in M. tuberculosis, which has a $\sim 65 \%$ $\mathrm{GC}$ content. This results in gaps in coverage and lower confidence for certain regions.

Sequencing technologies that generate longer reads include the Pacific Biosciences (PacBio) and Roche 454 sequencers. Long reads can substantially mitigate many of the issues above. PacBio single-molecule, real-time (SMRT) sequencing is also less affected by GC bias $^{178}$. Long reads can be combined with short reads to produce improved hybrid assemblies ${ }^{179}$. Sufficient sequencing depth with PacBio has also allowed the generation of high-quality complete genome sequences ${ }^{180}$. The primary limitations with long-read sequencers are costs (for both instruments and reagents), complexity and availability. Access to these technologies is thus still restricted.

Despite these issues, WGS enables molecular epidemiological studies to be carried out at an unrivalled resolution. WGS has allowed the retrospective differentiation between relapse and reinfection, the reconstruction of transmission chains, and the prediction of super-spreaders and potentially undiagnosed cases $^{181-183}$. Sequencing technologies are advancing at a rapid pace. With time, these advances hold promise to overcome current technical limitations. Moreover, as the cost of reagents and instruments continues to decrease, the opportunities for applying sequencing on the front line of the tuberculosis fight will only expand.

tree of the MTBC species that began to overturn prior beliefs about the origins of tuberculosis ${ }^{13}$ (FIG. 1).

In contrast to the notion that human tuberculosis arose as a zoonosis from domesticated cattle, the phylogeny revealed that animal strains of $M$. bovis are nested within a tree of human-adapted tuberculosis strains and thus represent derivative species relative to $M$. tuberculosis $^{13,14}$ (FIG. 1). This new phylogeny also casts doubt on the idea of a recent origin for human tuberculosis. The original zoonotic theory of $M$. tuberculosis posited that 


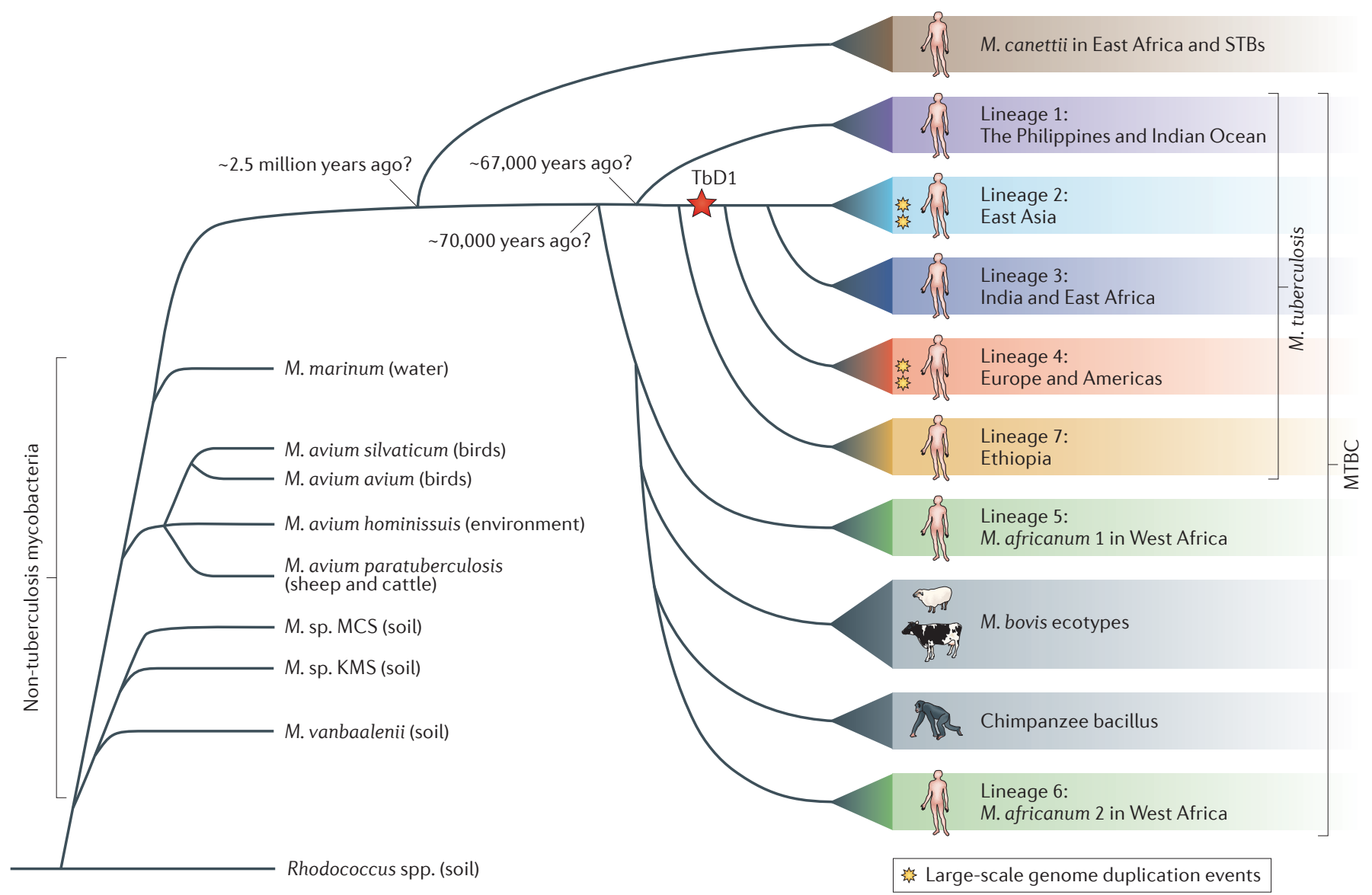

Figure 1 | Evolutionary relationship between selected mycobacteria and members of the Mycobacterium tuberculosis complex. Mycobacteria are predominantly environmental organisms. Several transitions from the environment to pathogenicity have occurred in these mycobacteria, including transitions for Mycobacterium avium and the Mycobacterium tuberculosis complex (MTBC). The MTBC was thought to arise as a clonal expansion from a smooth tubercle bacillus (STB) progenitor population. The animal-adapted Mycobacterium bovis ecotypes branch from a presumed human-adapted lineage of Mycobacterium africanum that is currently restricted to West Africa.

Human-adapted $M$. tuberculosis strains are grouped into seven main lineages, each of which is primarily associated with distinct geographical distribution. Colour coding for lineages corresponds to the colours in FIC. 2a,b. The dates of branching events are only crude estimates. TbD1 indicates the deletion event specific for $M$. tuberculosis lineages 2, 3 and 4. Evolutionary distances are not to scale. Detailed phylogenetic trees are provided in Supplementary information S2 (figure). All species shown are from the genus Mycobacterium.

Molecular clock

A method for dating

divergence between species based on the hypothesis of a constant rate of molecular change over time in selectively neutral DNA sequences.

Clonal expansion A population that arises from a single cell through cell replication without lateral gene transfer. human tuberculosis arose with the domestication of animals $\sim 10,000$ years ago. The new phylogeny predicted that animal strains instead diverged from human strains much earlier.

The notion of an older origin of M. tuberculosis from other human-infecting mycobacterial species was further supported by genomic studies of STBs, which are primarily associated with human tuberculosis in East Africa ${ }^{15-17}$. Initial studies suggested that STB isolates and, particularly, Mycobacterium canettii represent an early branching lineage of human-infecting mycobacteria ${ }^{13,15,18}$ (FIG. 1). This phylogenetic relationship was recently confirmed by WGS of five STB isolates ${ }^{19}$. This relationship was also used to attempt to date the origins of human tuberculosis. As both STB and M. tuberculosis strains are associated with human tuberculosis, parsimony can be used to argue that the last common ancestor of the two strains was also adapted to humans ${ }^{18}$. However, this assertion requires caution, as arguments for an environmental reservoir for STB strains have also been put forward ${ }^{20}$. Using a molecular clock analysis, one study suggested that the ancestor of $M$. tuberculosis and STB strains might have existed up to 2.8 million years $\mathrm{ago}^{18}$. Assuming that there was a human-adapted ancestor, this leads to the controversial hypothesis that mycobacterial disease in hominids may predate that in Homo sapiens and may have plagued human ancestors as far back as Homo habilis.

The studies on STBs suggest that the emergence of pathogenicity was associated with a change in evolutionary tempo. STB strains are genetically diverse and show evidence of both recombination and lateral gene transfer (LGT; also known as horizontal gene transfer). By contrast, the MTBC seems to be a single clonal expansion 
from this progenitor population. MTBC strains display low genetic diversity and no unambiguous evidence of LGT. A similar pattern of a clonal expansion from a recombining progenitor population has also been observed in Mycobacterium avium, which is a nontuberculosis mycobacterium related to $M$. tuberculosis and includes both environmental and animal pathogen strains $^{21-23}$ (FIG. 1). This shared pattern suggests potential common constraints in the evolution of pathogenicity within the mycobacteria. Clonality may be either an adaptation to or a consequence of pathogenicity, and it has implications for interpreting the evolution of mycobacteria. Low genetic diversity is consistent with pathogens emerging from a genetic bottleneck. The relative lack of sequence diversity within the MTBC, in particular, is consistent with a bottleneck at the split between the MTBC and the progenitor population.

The degree of genetic diversity within the MTBC has been a topic of debate. Early studies based on selected genes revealed little DNA diversity and led to the notion that strain variability in $M$. tuberculosis was negligible and clinically unimportant ${ }^{24,25}$. Subsequent studies using a larger number of markers called into question this conclusion and suggested the presence of genetic differences between $M$. tuberculosis strains from different parts of the world. The full breadth of such diversity was first revealed by the WGS of $25 \mathrm{M}$. tuberculosis strains that were selected to span the emerging genetic diversity ${ }^{20,26}$. The subsequent sequencing and analysis of an additional 229 strains provided an even more detailed view ${ }^{27}$. The comprehensive picture afforded by WGS has revealed substantially more genetic diversity than previously appreciated, and two strains of $M$. tuberculosis were found to differ by up to 2,000 single-nucleotide polymorphisms (SNPs) - a genetic distance that is comparable to the interspecies distance between $M$. tuberculosis and $M$. bovis ${ }^{20}$. Although this diversity is substantially smaller than that seen in other bacterial species (see below), there is an increasing amount of evidence that it may nonetheless have functional and perhaps clinical consequences $^{28}$.

WGS has also enabled the construction of the most reliable phylogenetic tree of MTBC strains so $\operatorname{far}^{27}$. Within this phylogeny seven major lineages of human pathogenic M. tuberculosis and M. africanum can be discerned along with a lineage of the animal-adapted strains of M. bovis (FIG. 1; see Supplementary information S2 (figure)). As previously detected using genotyping and selected sequencing ${ }^{29-31}$, each lineage is associated with a restricted geographical distribution ${ }^{32}$.

An updated model of M. tuberculosis origins. From the combination of archaeological, sequencing and comparative genomic analyses, a plausible scenario of the evolutionary origins of $M$. tuberculosis can be proposed (FIG. 2). Building on previous proposals ${ }^{13,29,33}$, components of this scenario have been described ${ }^{20,27,32,34,35}$. The deepest origins are most likely to lie in ancestral species that were adapted to soil and other environmental niches ${ }^{6,14,36}$. From these primordial roots, several transitions of mycobacteria to animal pathogenesis occurred.
For M. tuberculosis, this transition began within an STB progenitor population ${ }^{18,19}$. It remains unclear whether human pathogens arose in this population (and if so, when this happened). From this early population, a successful clone emerged to seed the MTBC. The argument that this emergence occurred in Africa ${ }^{32}$ is supported by the geographical restriction of $M$. canettii and other STBs to the Horn of Africa, the restriction of M. africanum to West Africa and the occurrence in Africa of all seven lineages of human-infecting M. tuberculosis ${ }^{32}$ (FIG. 1). A coalescence analysis of genome sequences from 259 MTBC strains further supports this hypothesis and suggests that the MTBC arose at least 70,000 years ago $^{27}$. From this origin, $M$. tuberculosis probably spread with human migration out of Africa. An analysis of the MTBC phylogeny suggests an early dispersal of MTBC lineage 1 that is coincident with the initial migration of humans out of Africa around the Indian Ocean as early as 67,000 years ago. A second dispersal may then have occurred $\sim 46,000$ years ago, which is coincident with the second wave of human migration into the Middle East, Europe and Asia ${ }^{27}$. Early human migration over the Bering Strait may provide an explanation for the archaeological evidence of tuberculosis in the ancient Americas (BOX 1). In addition, MTBC lineage 7 seems to have diverged after the primary migration out of Africa. MTBC lineage 7 is so far associated strictly with the Horn of Africa ${ }^{37}$ and may thus have arisen in a population that either stayed in or returned to Africa ${ }^{27}$. Sometime during this period, a clone of $M$. tuberculosis that was originally adapted to cause human tuberculosis evolved to infect a non-human mammal and thus began the transition into non-human ecotypes (for example, M. bovis). Such infections then spread to other animals, including cattle, goats, oryx and seals ${ }^{38-40}$. The early branching of the $M$. bovis clade suggests that its emergence predates animal domestication, although it has been noted that domestication may have contributed to the spread to livestock ${ }^{14}$. After M. tuberculosis emerged in Africa, its spread with human migration to early population centres in Western Europe, northern India and East Asia would provide an explanation for the early emergence of three main lineages of $M$. tuberculosis and for the archaeological evidence of tuberculosis in these regions (BOX 1; FIG. 2a). Human population expansion could have resulted in a concurrent expansion of these three lineages. Consistent with a co-divergence of MTBC and modern humans ${ }^{41}$, a comparison of the MTBC phylogeny with a tree constructed from human mitochondrial genomes reveals congruence ${ }^{27}$. Global exploration, trade, conquest and migration, coupled with the rapid growth in human population in modern times, would then have resulted in the worldwide spread of these initial lineages and led to the current phylogeographical distribution of tuberculosis (FIG. 2b).

Many questions remain concerning this hypothesized scenario. A recent analysis of $63 \mathrm{M}$. tuberculosis genomes supported the concurrent expansion of the $M$. tuberculosis and human populations but failed to find evidence of co-divergence between population structures ${ }^{42}$. The recent isolation of an $M$. tuberculosis strain from a wild 
a Before AD 1200

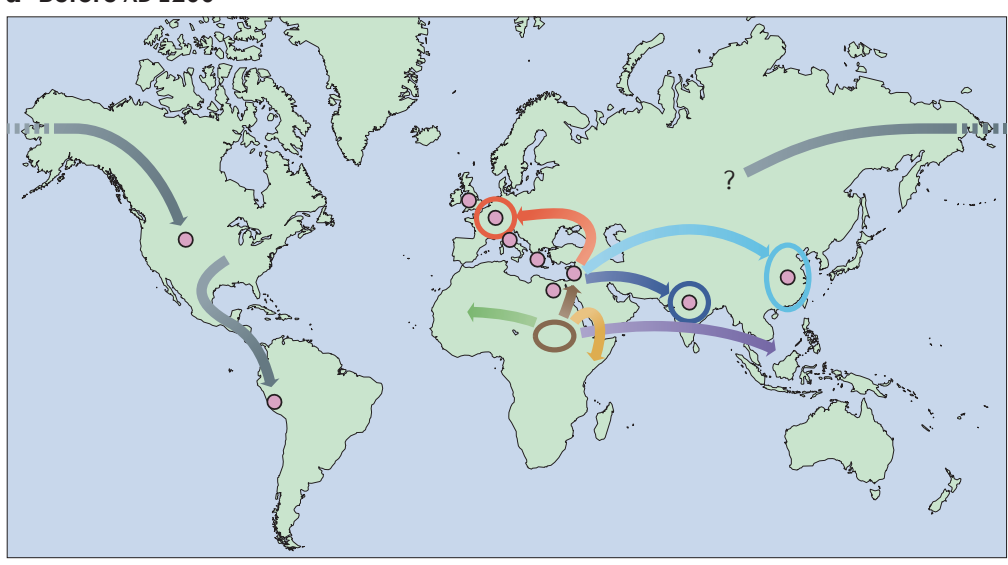

b After AD 1200

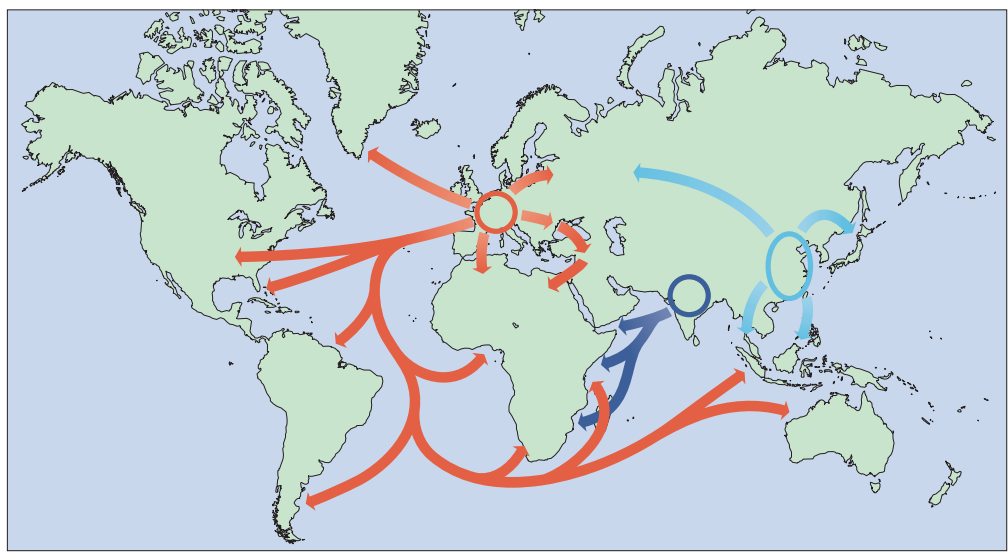

Figure 2 | Hypothesized evolutionary scenario for Mycobacterium tuberculosis and selected archaeological evidence. a | Early evolutionary events are shown. Colour coding for geographical locations corresponds to the lineages in FIG. 1. Mycobacterium tuberculosis and the M. tuberculosis complex (MTBC) arose from a smooth tubercle bacillus (STB) progenitor population in Africa through infections of early hominids. Mycobacterium africanum strains spread to West Africa (green arrow). During this period a strain evolved from $M$. africanum to infect non-human mammals. MTBC strains followed human migration from Africa to the Middle East and Asia (brown, purple, orange, yellow, light blue and dark blue arrows), which gave rise to documented archaeological evidence (pink circles). Potential migration across the Bering Strait may also have spread MTBC to the Americas (grey arrows). $\mathbf{b}$ | The spread of $M$. tuberculosis during modern times is shown. Human population expansions in Western Europe, northern India and East Asia gave rise to concurrent expansion of M. tuberculosis lineages in these regions. Global migration then resulted in the observed geographical distribution of these lineages. $\mathbf{c} \mid$ A timeline of evolutionary events and archaeological data is shown. The location for archaeological evidence is indicated in each box and corresponds to the pink circles in part $\mathbf{a}$. Boxes outlined in black indicate morphological evidence only, whereas boxes outlined in red denote both morphological and molecular evidence. A complete table of citations of archaeological evidence for tuberculosis in the ancient world is provided in Supplementary information S1 (table). M. bovis, Mycobacterium bovis; MYA, million years ago. Parts a and $\mathbf{b}$ are modified from REF. 32.

c

\section{Wyoming, USA \\ MTBC strain isolated from \\ Bison metacarpal remains}

\section{Egypt}

Extensive archaeological and molecular evidence of widespread human MTBC in mummies

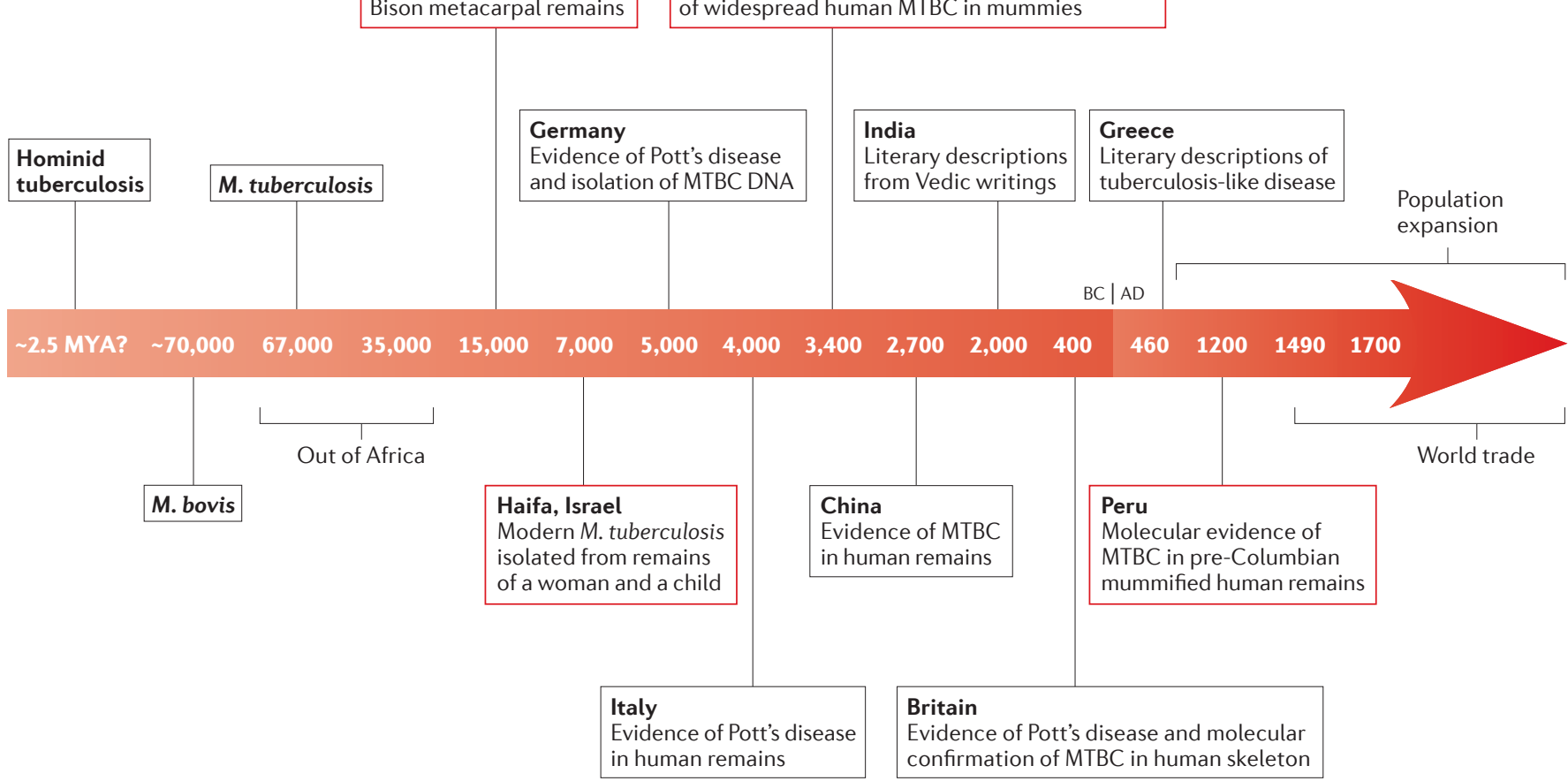


chimpanzee in Côte d'Ivoire also complicates the picture $^{43}$. WGS and phylogenetic analyses indicate that this strain clusters most closely with the M. africanum 2 lineage rather than with the M. bovis clade. However, several lines of evidence suggest that this strain in chimpanzees was not a result of infection from humans. Moreover, two other strains that were adapted to African mongoose and hydraxes have also been reported to cluster with the M. africanum 2 lineage $e^{44,45}$ but seem to be distinct from the strain in chimpanzees ${ }^{43}$. These interesting strains may imply that the earliest MTBC ancestor had a host range that was wider than the Homo genus. Alternatively, such strains may represent transitions of $M$. tuberculosis from humans to mammalian hosts that were distinct from the M. bovis transition. Further study into the full genetic and host diversity of the MTBC is required to answer these questions. Their answers, in turn, have important implications for understanding the nature of the co-evolution of M. tuberculosis and humans, the length of time over which this has occurred and the nature of $M$. tuberculosis pathogenesis, and therefore for the development of drugs and vaccines. In addition, the mechanisms of evolution have an immediate and important consequence for understanding the most recent evolutionary adaptation of $M$. tuberculosis: the emergence of drug resistance.

\section{Recent evolution and drug resistance}

Until $\sim 80$ years ago, there was no drug to treat tuberculosis. Since then, we have witnessed the emergence of single-drug resistant, multiple-drug resistant (MDR) and extensively drug resistant (XDR) M. tuberculosis strains. Most recently, four different countries have reported strains that are resistant to all drugs tested, which are known as totally drug resistant (TDR) M. tuberculo$s i s^{46-49}$. Drug resistance is a widespread problem in all bacterial pathogens and is typically driven by a combination of LGT, genome rearrangement and nucleotide mutation. By contrast, evolution of M. tuberculosis seems to be more restricted, which raises challenges for understanding resistance in tuberculosis.

Genetic mechanisms and rates of recent evolution. LGT has long been considered a 'driver' for the evolution of new gene functions. In contrast to other bacteria ${ }^{50-54}$, evidence of LGT had been lacking within the MTBC. Comparative genomics between $M$. tuberculosis and related mycobacteria have provided strong evidence for the role of gene gains and losses in the evolution of mycobacterial ancestors ${ }^{55-59}$, and the progenitor population of STBs and M. tuberculosis shows clear signs of recombination ${ }^{18,19}$. However, since the emergence of the MTBC from this progenitor population, MTBC evolution seems to be mostly clonal ${ }^{60,61}$. Consistent with this, only $1 \%$ of SNPs in 259 sequenced MTBC strains display evidence of homoplasy ${ }^{27}$. However, a recent comparative analysis ${ }^{62}$ has provided evidence for ongoing recombination, albeit for short tracts of DNA. Further work is needed to confirm these intriguing findings.

Similarly, until recently, large-scale genome rearrangements were generally considered to be absent from the MTBC. Studies of larger-scale polymorphisms reported predominantly deleted sequences of small genomic regions that span part of a gene to several genes $^{63-66}$. These data have led to the prevailing view that genome-scale evolution in the MTBC has mostly occurred by gene loss ${ }^{6}$. Such a pattern is often assumed to be consistent with reductive evolution that is associated with an intracellular pathogenic lifestyle ${ }^{36}$, and a similar pattern was observed in M. avium pathogenic strains relative to environmental strains ${ }^{67}$. However, two recent studies reveal that large-scale duplications are not altogether absent in M. tuberculosis ${ }^{68,69}$. WGS reported multiple large-scale duplications of the same genomic region in $M$. tuberculosis ${ }^{69}$. Originally identified only in the Beijing lineage ${ }^{68}$, WGS revealed such occurrences in multiple lineages with substantially different boundaries, which indicates independent originating events. The independent duplication of the same large genomic region in multiple strains suggests instability of this region and/or a selective advantage for these duplications. Along with the identification of a smaller segmental duplication in the laboratory strain H37Rv, these polymorphisms suggest that large-scale duplication events may be more common than previously considered. Gene duplication is an evolutionary driver of new gene functions $s^{50,52,54}$ and has been linked to both drug resistance and virulence in bacteria ${ }^{51,54,70,71}$. Thus, the results in $M$. tuberculosis warrant further study.

In spite of the evidence above, genome evolution in M. tuberculosis is thought to be mostly driven by sequential chromosomal nucleotide substitutions. Understanding the rate of nucleotide mutation in M. tuberculosis is thus central to our knowledge of the process of drug resistance evolution. To address this issue, one study carried out WGS on strains of $M$. tuberculosis isolated from cynomolgus macaques ${ }^{72}$. By sequencing both the infecting strain of $M$. tuberculosis and isolates after various times of infection, the authors were able to estimate the mutation rate in monkeys during various stages of tuberculosis infection. The estimate of $2 \times 10^{-10}$ mutations per cell division of $M$. tuberculosis for active disease places the mutation rate at the lower end of the spectrum for bacteria. More surprisingly, the mutation rate per unit time for latent disease, reactivated disease and M. tuberculosis grown in vitro was not significantly different from that for $M$. tuberculosis during active disease. A subsequent study also confirmed that these rates were similar to those estimated from the sequencing of human isolates ${ }^{73}$. The finding that the M. tuberculosis per-time mutation rate is similar between different stages of tuberculosis is controversial ${ }^{74}$ because much evidence suggests that $M$. tuberculosis replicates at substantially different rates during different stages ${ }^{75,76}$. During active disease, it is thought that bacteria may exist in subpopulations with heterogeneous phenotypes within a single patient and even within a single granuloma ${ }^{3}$. During latent disease, $M$. tuberculosis is thought to enter a dormant state in which the replication rate is substantially slower than that during active growth. In many bacteria, mutation rate is linked to errors during replication. On the bases of their results and particular 
mutations observed, the authors propose an alternative model for M. tuberculosis infection. Mutations during latent infection might be explained by oxidative damage in the host. The mutation rate would then be linked to the amount of time growing in the host rather than to replication rate. The role of oxidative stress in the development of drug resistance has also been recently described in Escherichia coli ${ }^{77-79}$.

Implications of mutation rates for the emergence of drug resistance. The low mutation rate is consistent with the lack of genetic diversity among $M$. tuberculosis strains but is surprising in the face of the rapid emergence of multiple-drug resistance and the increasing amount of evidence for the diversity of M. tuberculosis in vivo. WGS of serial patient isolates has documented that resistance mutations to many drugs are often independently acquired several times by different isolates ${ }^{80-83}$. In the case of one patient who was infected with an M. tuberculosis Beijing strain ${ }^{82}$, different drug resistance mutations were found at different times during a nine-year period. Moreover, the evidence suggested that multiple different resistance mutations transiently existed within this patient between selective sweeps. A subsequent study confirmed this possibility by using WGS to directly characterize the genetic diversity of the M. tuberculosis population in individual patients ${ }^{84}$. Rather than isolating single bacterial colonies for sequencing, one study cultured bacteria from sputum samples and sequenced all colonies that were present. By sequencing to a high depth of coverage, the researchers could identify mutations at specific loci that are present only in a proportion of the bacteria sequenced. Using this approach, they confirmed the surprising heterogeneity of mutations in a single patient, albeit in sputa: as many as $4-5$ different resistance mutations for a single drug could be detected, although only one was ultimately fixed in the population. Resistance to a single drug can thus arise multiple times, even in an individual patient ${ }^{85}$.

Together, these data have raised the question of whether the slow mutation rate of M. tuberculosis is sufficient to provide an explanation for the relatively rapid rate of multiple-drug resistance acquisition observed ${ }^{86}$. Several factors may play a part in this question ${ }^{74}$. One factor may be phenotypic: it has been proposed that transient hypermutator phenotypes might arise, as seen in other bacteria ${ }^{87}$. In M. tuberculosis, deletion of the error-prone DNA polymerase (DnaE2) reduced the rate of rifampicin resistance acquisition during treatment in mice ${ }^{88}$. This led to the suggestion that changes in the expression of DnaE2 alter the rate of resistance acquisition. Another important factor seems to be the genetic diversity in the different $M$. tuberculosis lineages. Experimental and epidemiological evidence has long suggested that different strains of $M$. tuberculosis can vary in their propensity to develop drug resistance. In particular, the Beijing family of strains that belong to the East Asian lineage has been associated with an increased rate of acquiring resistance mutations ${ }^{89-92}$. The relative contribution of bacterial genetic background and other

epidemiological factors to this association has been unclear. One study showed that $M$. tuberculosis strains from different lineages differed in their intrinsic mutation rates: strains from the East Asian lineage acquired drug resistance in vitro more rapidly than strains from the Euro-American lineage ${ }^{73}$. These differences in mutation rates are consistent with known mutations in DNA replication and repair genes in strains from the East Asian lineage ${ }^{93}$. These data imply that patients infected with strains from different lineages are at different risks for developing drug-resistant disease. Such a possibility requires connecting the measured mutation rates with observed rates of drug resistance acquisition during treatment, and has substantial implications for treatment strategies.

In addition to the acquisition of de novo drug resistance, molecular epidemiological studies (BOX 2) have also highlighted the importance of the transmission of strains that are already resistant (that is, primary resistance). The frequency at which resistant strains are transmitted has been a topic of debate ${ }^{94}$. It was once assumed that such strains would suffer a fitness cost relative to susceptible strains in the absence of treatment. However, several studies revealed that many resistance mutations carried no fitness costs and that these are the most common mutations in clinical isolates ${ }^{95-100}$. Moreover, the fitness cost of deleterious resistance mutations can be offset by compensatory mutations ${ }^{95,100}$. For example, a WGS study confirmed that mutations in rpoA and rpoC (which encode the $\alpha$ and $\beta^{\prime}$ subunits of RNA polymerase, respectively) seem to offset the fitness cost of mutations in $r p o B$ (which encodes the $\beta$ subunit of RNA polymerase) that lead to rifampicin resistance ${ }^{101,102}$. These compensatory mutations are observed in a large proportion of clinical isolates ${ }^{83,101}$. These data imply that resistant strains may persist in the global $M$. tuberculosis population even in the absence of drug treatment.

\section{From genomes to function: systems biology}

Beyond mapping genome sequence and structure, which is rapidly becoming routine, sequencing-based technologies are now also enabling global profiling of genome function. The combination of genomics with other 'omics' technologies - such as proteomics, metabolomics and lipidomics - has the ability to assay cells with unprecedented breadth and depth, as well as across a range of timescales and experimental contexts. Thus, there is the potential to develop coherent models of the systems themselves that can be used to predict and understand dynamic system behaviour in a range of contexts.

A recent report described the application of this approach for understanding the molecular systems that underlie the ability of $M$. tuberculosis to survive in the host ${ }^{103}$. At the heart of this effort was the use of chromatin immunoprecipitation followed by sequencing (ChIPseq) ${ }^{104-107}$ for the characterization of the genome-wide occupancy of M. tuberculosis transcription factors to delineate the global transcription factor regulatory network. The Tuberculosis Systems Biology Consortium was the first to apply ChIP-seq on a large scale to globally map transcription factor binding sites in bacteria ${ }^{103}$.
Reductions in genetic variation due to strong selection for advantageous alleles. 
The consortium recently reported a genome-scale map of the regulatory interactions of 50 transcription factors (which constitute $26 \%$ of predicted $M$. tuberculosis transcription factors), and many more have since been completed (see the Tuberculosis Database).

The M. tuberculosis ChIP-seq data have confirmed several surprises that have also emerged from extensive ChIP-seq mapping of transcription factors in nearly all other organisms ${ }^{107}$. This confirmation calls into question some of the simplifying assumptions about bacterial transcriptional regulation, as reviewed elsewhere ${ }^{107,108}$. First, the data are revealing that binding of transcription factors in $M$. tuberculosis occurs in many more diverse genomic locations than simply the promoter-proximal regions that are expected on the basis of the canonical model of regulation. ChIP-seq data from M. tuberculosis confirm that binding in upstream intergenic regions is enriched over that expected by chance, but this represents less than $40 \%$ of the binding events for any transcription factor. The majority of binding events occur outside upstream intergenic regions in either genic or converging intergenic regions, which suggests a potentially more complex role for transcription factor binding ${ }^{107,109}$. Second, mapping in all organisms has revealed many more binding sites even for well-studied regulators $^{109}$, and this was also true for M. tuberculosis ${ }^{103,107}$. The functions of most of these novel binding sites in all organisms remain unknown. In M. tuberculosis these novel binding sites are nearly always associated with an underlying sequence motif, but not all instances of motifs are bound. Depending on the transcription factor, less than $40-70 \%$ of potential binding sites are occupied in vivo. Thus, $M$. tuberculosis transcription factor binding is specific to genomic context ${ }^{103}$. The determinants of this specificity remain unknown.

The binding data also suggest that the M. tuberculosis regulatory network is far more complex and interconnected than previously assumed. For example, there is substantial binding between transcription factors (FIG. 3a). This complexity mirrors that seen in regulatory networks in other bacteria and eukaryotes ${ }^{110-112}$, and its implications have been the topic of intense study. One key finding is that this connectivity results in many nested feedback and feedforward loops (known as network motifs) that are known to give rise to nontrivial expression dynamics, including gene expression pulses ${ }^{111,113,114}$. Such dynamics, which are typically invisible to steady-state or single time-point expression measurements ${ }^{107}$, are essential for understanding complex cellular behaviour ${ }^{51,52,70,115,116}$.

The importance of expression dynamics in M. tuberculosis has recently been demonstrated by an investigation of the response of single cells to treatment with the antibiotic isoniazid (INH) ${ }^{117}$. Antibiotic treatment of $M$. tuberculosis and other bacteria typically only kills a proportion of cells, and this persistence in the face of antibiotics is thought to contribute to the difficulties of effectively treating tuberculosis and possibly to the emergence of drug resistance. Using a microfluidic device and time-lapse microscopy, one study showed that the catalase-peroxidase-peroxynitritase $\mathrm{T}($ Kat $G)$ gene that activates INH is expressed in stochastic pulses. These pulses were only present in a minority of cells. Moreover, pulsing was negatively correlated with cell survival after treatment with INH. These data suggest that infrequent pulsatile expression of $\mathrm{Kat} G$ has a role in allowing $M$. tuberculosis to adapt to drug exposure. The mechanisms that generate $K a t G$ pulsing are not yet known. The considerations above suggest that aspects of the complexity of the regulatory network mapped at a global level may well explain this behaviour, which is only visible when $M$. tuberculosis is observed at a highly granular level.

The regulatory network map also begins to reveal interactions between transcription factors that mediate the complex and dynamic responses of $M$. tuberculosis to the host environment (FIG. 3a), such as the hypoxic conditions within macrophages. For example, the data show an interconnected subnetwork that links hypoxic adaptation, lipid and cholesterol degradation, and lipid biosynthesis. These processes, which are among the most extensively studied in $M$. tuberculosis, are often treated as separate, disconnected phenomena. However, they are linked biochemically ${ }^{118}$, and the emerging $M$. tuberculosis regulatory network reveals that they may also be linked in terms of gene regulation (FIG. 3a). Consistently, a systems-level profiling of lipids, proteins, metabolites and mRNA in M. tuberculosis during a time course of hypoxia and subsequent re-aeration in vitro uncovered numerous alterations in lipid content, as well as changes in gene expression and metabolites in corresponding metabolic pathways ${ }^{103}$. For example, changes in oxygen tension produced rapid and reversible changes in the expression of genes that are necessary for cholesterol degradation. This was surprising because although cholesterol is present in host cells, the culture conditions used for profiling contained no cholesterol, and $M$. tuberculosis is unable to synthesize cholesterol de novo. These and other changes suggest that changes in oxygen levels evoke a regulatory and metabolic programme that results in changes which are specifically adapted to the host environment. This hypothesis, in turn, is consistent with the emerging picture of $M$. tuberculosis as a pathogen that has evolved over a substantial period of time to interact specifically with the human immune system.

\section{The host-pathogen interaction}

A successful $M$. tuberculosis infection is a delicate balance. On the one hand, M. tuberculosis requires an immune response that is adequate to establish a granuloma. However, the immune response must not be substantial enough to lead to complete sterilization. On the other hand, inflammation over time must be sufficient to promote the eventual breakdown of some granulomas for transmission. The growing evidence suggests that striking this balance requires a complex programme of cellular changes in both the pathogen and the host that is partly induced and coordinated by M. tuberculosis.

The mechanisms underlying these changes are many, complex and still poorly understood. However, there is an increasing amount of evidence that part of the 

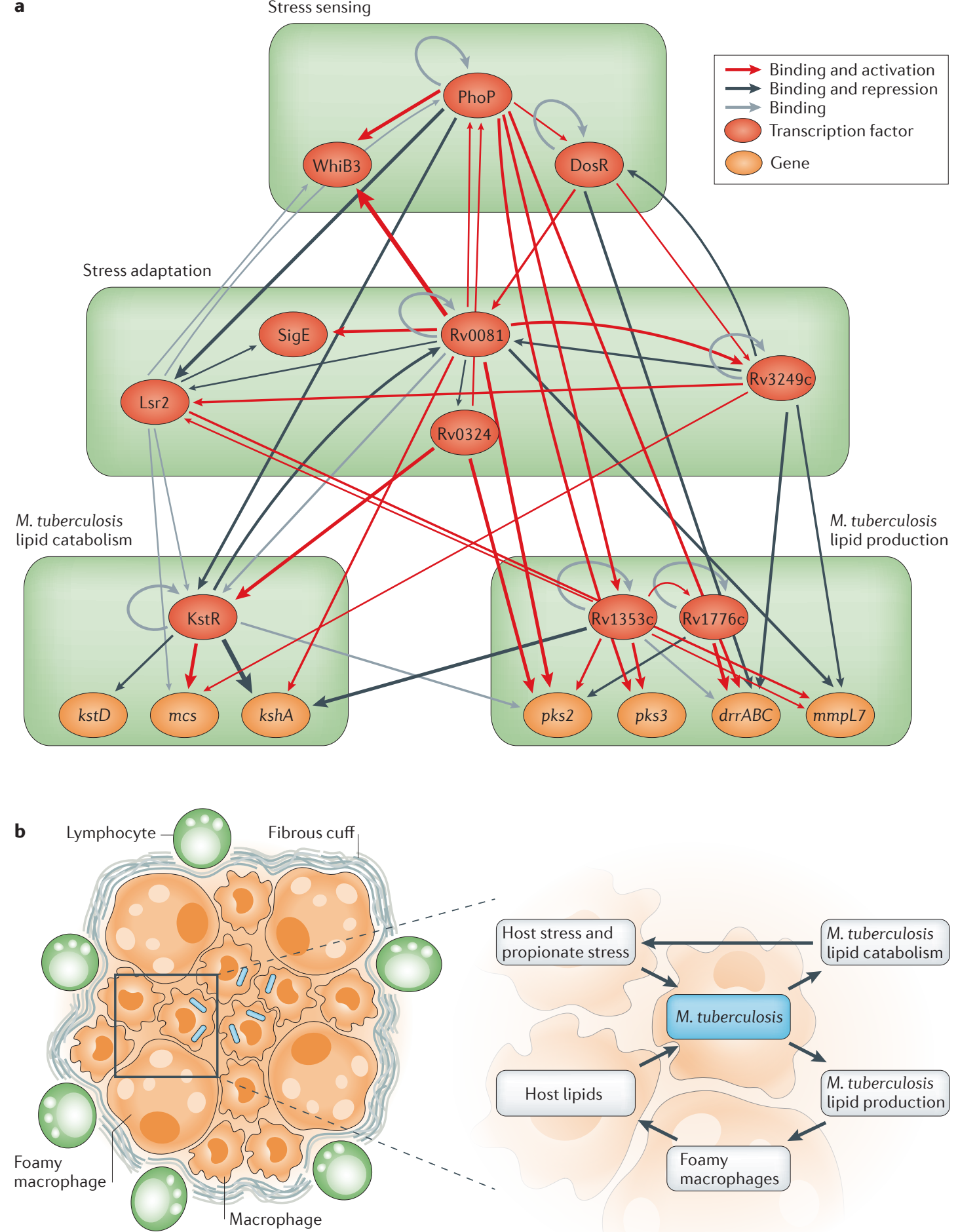

Figure 3 | Host-pathogen interactions. a | Selected regulatory interactions that link responses to stress with changes in lipid metabolism are shown. Interactions were identified by chromatin immunoprecipitation followed by sequencing (ChIP-seq), transcription factor perturbation and transcriptomics ${ }^{103}$. Links are represented by arrows, the colours of which indicate predicted regulatory effects of the transcription factors on the target genes. $\mathbf{b} \mid$ A simplified model of the interactions between Mycobacterium tuberculosis and the host in an infected granuloma is shown. Interactions include two hypothesized feedback loops that are mediated partly by the regulatory interactions in part a. Host stresses induce metabolic changes in M. tuberculosis, which lead to the catabolism of host lipids (including cholesterol). Degradation of cholesterol leads to propionate build-up in the bacterium, which can be alleviated by the assimilation of other host lipids into the production of immunomodulatory lipids. Certain immunomodulatory lipids induce the formation of foamy macrophages that enclose numerous intracellular lipid-containing bodies. Lipids from these bodies can be accessed by M. tuberculosis. Thus, M. tuberculosis responds to the host environment and the digestion of host lipids, leading to the production of immunomodulatory lipids that shape the host environment to increase the availability of host lipids. 
explanation involves interactions that lead to changes in lipid metabolism in both the pathogen and the host (FIG. 3b). According to this scenario, host stresses ${ }^{119,120}$ induce metabolic changes in $M$. tuberculosis that include a switch to the catabolism of host lipids, particularly cholesterol ${ }^{19,121-125}$. Alterations in M. tuberculosis lipid metabolism are tightly linked with changes in the production of toxic propionate, the detoxification of which is central to M. tuberculosis host survival ${ }^{126}$. This programme also results in alterations in immunomodulatory cell wall lipids that can be trafficked through macrophages ${ }^{103,127,128}$ and induce host changes, including the conversion of macrophages to foam cells that have numerous lipid-containing bodies ${ }^{129,130}$. The lipids in these bodies, which include cholesterol, can be accessed by $M$. tuberculosis. Alterations in M. tuberculosis metabolism, including the digestion of host cholesterol, lead to propionate build-up ${ }^{121}$, which can be alleviated in $M$. tuberculosis by the assimilation of host lipids into immunomodulatory cell wall lipids ${ }^{118,131,132}$. This completes the loop through the stimulation of further host cell lipid availability. In short, host cells and M. tuberculosis may interact partly through positive feedback loops, in which responses to the host environment and the digestion of host lipids lead to the production of M. tuberculosis immunomodulatory lipids that shape the host environment to increase the availability of host lipids. This scenario is consistent with the regulatory links discovered in the $M$. tuberculosis regulatory network described above.

These and other interactions characterize $M$. tuberculosis as a pathogen that has evolved specific cellular programmes that not only respond to the host environment but also orchestrate changes to it ${ }^{3,132-136}$. One of the most compelling pieces of evidence that support this view was derived from the sequencing of strains spanning the genetic diversity of $M$. tuberculosis described above. A common theme for many human pathogens is that genes encoding antigens are frequently highly variable to allow pathogens to escape detection by the immune system. However, an analysis revealed that the situation in M. tuberculosis seems to be different ${ }^{26}$. The authors examined the conservation of all genes between the sequenced $M$. tuberculosis strains. As expected, genes that are known to be essential were more conserved than non-essential genes. Surprisingly, the majority of known $\mathrm{T}$ cell antigen-encoding genes were also as conserved as essential genes. Moreover, the epitope-encoding regions within these genes were the most conserved regions of these or any genes. This apparent purifying selection of $\mathrm{T}$ cell antigens has since been confirmed by a comparative analysis of sequenced $M$. tuberculosis and STB strains ${ }^{19}$. These results do not include data on PE-PPE genes, which constitute a highly repetitive antigenic gene family that is typically not captured by shortread sequencing ( $\mathrm{BOX} 2$ ). Moreover, more work is required to link specific immune responses to the predicted conserved antigens. Nonetheless, these remarkable data suggest that, unlike other pathogens that seek to avoid host immune responses, $M$. tuberculosis is potentially under selective pressure to instigate such a response $\mathrm{e}^{26}$.

\section{Conclusions}

The reports documented in this Review give rise to a picture of M. tuberculosis as a pathogen that is remarkably adapted for life in human host cells, and the evolutionary history of this bacterium suggests that this adaptation may have evolved over thousands, if not millions, of years. This alters our perspective on the disease. As previously noted, the formation of a tuberculosis granuloma is generally thought to be a response of the immune system to the bacterium, whereas breakdown of the granuloma then represents a failure of this response ${ }^{3}$. However, from the perspective of long-term evolution of $M$. tuberculosis with humans, the granuloma can also been seen as an adaption of $M$. tuberculosis to enable a persistent infection, which is a likely requirement in the face of low human population densities during pre-history ${ }^{3,137}$. The subsequent expansion of the human population has altered this dynamic, and more recent evolutionary events may be driving additional changes. Co-infection with both $M$. tuberculosis and HIV amplifies the impact on the host immune system and thus the effects of both tuberculosis and AIDS $^{138}$. The impact of these and other recent events (for example, the emergence of diabetes ${ }^{139}$ ) on the evolution of $M$. tuberculosis remains to be seen.

This picture of host-pathogen interactions has implications for vaccine development efforts. The challenge facing immunologists is the development of a vaccine against an organism in which infection with the entire organism is insufficient to generate protective immunity. This challenge is illustrated by the failure of vaccination with the attenuated bacille CalmetteGuérin (BCG) strain to induce lifelong immunity. Such a challenge is potentially compounded by the finding that many of the antigens being used to develop vaccines seem to be under strong purifying selection ${ }^{26}$. As noted by a previous study, this is a double-edged sword: the lack of diversity may simplify vaccine development, but the possibility that the immune response induced by most antigens may have evolved to benefit M. tuberculosis complicates efforts ${ }^{26}$.

Encouragingly, the expanding application of genomics is providing new means for combating this old foe. Although most antigens seem to be highly conserved, sequencing has revealed a small subset of antigens that do show variation ${ }^{26}$. These may thus warrant further study in the context of vaccine discovery. The widespread sequencing and analyses of $M$. tuberculosis strains are also providing new insights into the ongoing evolution of $M$. tuberculosis during infection, treatment and the acquisition of drug resistance ${ }^{85}$. These results can also be used to develop more effective strategies for deploying existing drugs, such as by analysing drug resistance mutations in patient-derived populations of $M$. tuberculosis to predict the drugs that might be most clinically effective for a particular patient. Finally, systems mapping is uncovering the complex regulatory programmes that have evolved to enable the organism to survive in the host ${ }^{103}$. Disruptions of these programmes may thus represent new avenues for drug discovery. 
1. World Health Organization Global Tuberculosis Control (WHO, 2010)

2. Russell, D. G. Mycobacterium tuberculosis: here today, and here tomorrow. Nature Rev. Mol. Cell Biol. 2, 569-577 (2001)

3. Russell, D. G. Who puts the tubercle in tuberculosis? Nature Rev. Microbiol. 5, 39-47 (2006).

4. Kaufmann, S. H. E. How can immunology contribute to the control of tuberculosis? Nature Rev. Immunol. 1 20-30 (2001)

5. Parrish, N. M., Dick, J. D. \& Bishai, W. R. Mechanisms of latency in Mycobacterium tuberculosis. Trends Microbiol. 6, 107-112 (1998).

6. Veyrier, F. J., Dufort, A. \& Behr, M. A. The rise and fall of the Mycobacterium tuberculosis genome. Trends Microbiol. 19, 156-161 (2011).

7. Kapur, V., Whittam, T. S. \& Musser, J. M. Is Mycobacterium tuberculosis 15,000 years old? J. Infect. Dis. 170, 1348-1349 (1994).

8. Bates, J. H. \& Stead, W. W. The history of tuberculosis as a global epidemic. Med. Clin. North Am. 77 , 1205-1217 (1993).

9. Cole, S. T. et al. Deciphering the biology of Mycobacterium tuberculosis from the complete genome sequence. Nature 393, 537-544 (1998); erratum 396, 190 (1998).

This is a groundbreaking report of the firs whole-genome sequence for $M$. tuberculosis.

10. Behr, M. A. et al. Comparative genomics of BCG vaccines by whole-genome DNA microarray. Science 284, 1520-1523 (1999).

11. Gordon, S. V. et al. Identification of variable regions in the genomes of tubercle bacilli using bacterial artificia chromosome arrays. Mol. Microbiol. 32, 643-655 (1999).

12. Brosch, R. et al. Genomic analysis reveals variation between Mycobacterium tuberculosis H37Rv and the attenuated $M$. tuberculosis H37Ra strain. Infect. Immun. 67, 5768-5774 (1999).

13. Brosch, R. et al. A new evolutionary scenario for the Mycobacterium tuberculosis complex. Proc. Natl Acad. Sci. USA 99, 3684-3689 (2002). This analysis of genomic deletions reveals that $M$. bovis and related animal-adapted ecotypes are probably derived from human-adapted $M$. tuberculosis, which reverses the prevailing view that $M$. tuberculosis is a zoonosis derived from domesticated animals.

14. Mostowy, S. \& Behr, M. A. The origin and evolution of Mycobacterium tuberculosis. Clin. Chest Med. 26 207-216 (2005).

15. van Soolingen, D. et al. A novel pathogenic taxon of the Mycobacterium tuberculosis complex, Canetti characterization of an exceptional isolate from Africa. Int. J. Syst. Bacteriol. 47, 1236-1245 (1997).

16. Koeck, J. L. et al. Clinical characteristics of the smooth tubercle bacilli 'Mycobacterium canettii' infection suggest the existence of an environmental reservoir Clin. Microbiol. Infect. 17, 1013-1019 (2011).

17. Fabre, M et al. Molecular characteristics of "Mycobacterium canettii" the smooth Mycobacterium tuberculosis bacilli. Infect. Genet. Evol. 10 1165-1173 (2010)

18. Gutierrez, M. C. et al. Ancient origin and gene mosaicism of the progenitor of Mycobacterium tuberculosis. PLoS Pathog. 1, e5 (2005) This is the first report to indicate that STBs are an early branching lineage of the MTBC, which leads to the controversial suggestion that human tuberculosis-causing mycobacteria may have evolved up to 2.8 million years ago.

19. Supply, P. et al. Genomic analysis of smooth tubercle bacilli provides insights into ancestry and pathoadaptation of Mycobacterium tuberculosis. Nature Genet. 45, 172-179 (2013).

20. Gagneux, S. Genetic diversity in Mycobacterium tuberculosis. Curr. Top. Microbiol. Immunol. 374 $1-25$ (2013)

21. Biet, F. et al. Inter- and intra-subtype genotypic differences that differentiate Mycobacterium avium subspecies paratuberculosis strains. BMC Microbiol. 12, 264 (2012).

22. Turenne, C. Y., Wallace, R. Jr \& Behr, M. A. Mycobacterium avium in the postgenomic era. Clin. Microbiol. Rev. 20, 205-229 (2007).

23. Turenne, C. Y., Collins, D. M., Alexander, D. C. \& Behr, M. A. Mycobacterium avium subsp. paratuberculosis and M. avium subsp. avium are independently evolved pathogenic clones of a much broader group of $M$. avium organisms. J. Bacteriol. 190, 2479-2487 (2008).
24. Sreevatsan, S. et al. Restricted structural gene polymorphism in the Mycobacterium tuberculosis complex indicates evolutionarily recent global dissemination. Proc. Natl Acad. Sci. USA 94, 9869-9874 (1997).

25. Musser, J. M., Amin, A. \& Ramaswamy, S Negligible genetic diversity of Mycobacterium tuberculosis host immune system protein targets: evidence of limited selective pressure. Genetics 155 , 7-16 (2000)

26. Comas, I. et al. Human T cell epitopes of Mycobacterium tuberculosis are evolutionarily hyperconserved. Nature Genet. 42, 498-503 (2010). This study analyses whole-genome sequences from $21 M$. tuberculosis strains and reveals that $\mathrm{T}$ cell antigens are as conserved as essential genes. These results suggest that $M$. tuberculosis antigens may have evolved to specifically interact with the human immune system.

27. Comas, I. et al. Out-of-Africa migration and Neolithic coexpansion of Mycobacterium tuberculosis with modern humans. Nature Genet. 45, 1176-1182 (2013)

This analysis of genome sequences from 259 MTBC strains has provided the most complete phylogeny of $M$. tuberculosis so far and supports a detailed scenario of the origins of human tuberculosis.

28. Coscolla, M. \& Gagneux, S. Does M. tuberculosis genomic diversity explain disease diversity? Drug Discov. Today Dis. Mech. 7, e43-e59 (2010).

29. Wirth, T. et al. Origin, spread and demography of the Mycobacterium tuberculosis complex. PLoS Pathog. 4, e1000160 (2008)

30. Brudey, K. et al. Mycobacterium tuberculosis complex genetic diversity: mining the fourth international spoligotyping database (SpolDB4) for classification, population genetics and epidemiology. BMC Microbiol. 6, 23 (2006).

31. Mathema, B., Kurepina, N. E., Bifani, P. J. \& Kreiswirth, B. N. Molecular epidemiology of tuberculosis: current insights. Clin. Microbiol. Rev. 19 658-685 (2006)

32. Hershberg, R. et al. High functional diversity in Mycobacterium tuberculosis driven by genetic drift and human demography. PLoS Biol. 6, e311 (2008)

33. Mostowy, S., Cousins, D., Brinkman, J., Aranaz, A. \& Behr, M. A. Genomic deletions suggest a phylogeny for the Mycobacterium tuberculosis complex. J. Infect. Dis. 186, 74-80 (2002)

34. Gagneux, S. \& Small, P. M. Global phylogeography of Mycobacterium tuberculosis and implications for tuberculosis product development. Lancet Infect. Dis. 7, 328-337 (2007)

35. Gagneux, S. et al. Variable host-pathogen compatibility in Mycobacterium tuberculosis. Proc. Natl Acad. Sci. USA 103, 2869-2873 (2006)

36. Behr, M. A. Mycobacterium du jour: what's on tomorrow's menu? Microbes Infect. 10, 968-972 (2008)

37. Firdessa, R. et al. Mycobacterial lineages causing pulmonary and extrapulmonary tuberculosis, Ethiopia. Emerg. Infect. Dis. 19, 460-463 (2013).

38. Garnier, T. et al. The complete genome sequence of Mycobacterium bovis. Proc. Natl Acad. Sci. USA 100. 7877-7882 (2003)

39. Prodinger, W. M. et al. Characterization of Mycobacterium caprae isolates from Europe by mycobacterial interspersed repetitive unit genotyping. J. Clin Microbiol. 43, 4984-4992 (2005).

40. Cousins, D. V. et al. Tuberculosis in seals caused by a novel member of the Mycobacterium tuberculosis complex: Mycobacterium pinnipedii sp. nov. Int. J. Syst. Evol. Microbiol. 53, 1305-1314 (2003).

41. Gagneux, S. Host-pathogen coevolution in human tuberculosis. Phil. Trans. R. Soc. B 367, 850-859 (2012).

42. Pepperell, C. S. et al. The role of selection in shaping diversity of natural $M$. tuberculosis populations. PLoS Pathog 9 e1003543 (2013).

43. Coscolla, M. et al. Novel Mycobacterium tuberculosis complex isolate from a wild chimpanzee. Emerg. Infect. Dis. 19, 969-976 (2013).

44. Cousins, D. V., Peet, R. L., Gaynor, W. T, Williams, S. N. \& Gow, B. L. Tuberculosis in imported hyrax (Procavia capensis) caused by an unusual variant belonging to the Mycobacterium tuberculosis complex. Vet. Microbiol. 42, 135-145 (1994).

45. Alexander, K. A. et al. Novel Mycobacterium tuberculosis complex pathogen, $M$. mungi. Emerg. Infect. Dis. 16, 1296-1299 (2010).
46. Udwadia, Z. \& Vendoti, D. Totally drug-resistan tuberculosis (TDR-tuberculosis) in India: every dark cloud has a silver lining. J. Epidemiol. Commun. Health 67, 471-472 (2013)

47. Migliori, G. B., De laco, G., Besozzi, G., Centis, R. ¿ Cirillo, D. M. First tuberculosis cases in Italy resistant to all tested drugs. Euro Surveill 12, E070517.1 (2007)

48. Klopper, M. et al. Emergence and spread of extensively and totally drug-resistant tuberculosis, South Africa. Emerg. Infect. Dis. 19, 449-455 (2013).

49. Velayati, A. A. et al. Emergence of new forms of totally drug-resistant tuberculosis bacilli: super extensively drug-resistant tuberculosis or totally drug-resistant strains in Iran. Chest 136, 420-425 (2009).

50. Ohno, S. Evolution by Gene Duplication (SpringerVerlag, 1970).

51. Sandegren, L. \& Andersson, D. I. Bacterial gene amplification: implications for the evolution of antibiotic resistance. Nature Rev. Microbiol. 7 , 578-588 (2009).

52. Ochman, H., Lawrence, J. G. \& Groisman, E. A. Lateral gene transfer and the nature of bacterial innovation. Nature 405, 299-304 (2000).

53. Boucher, Y. et al. Lateral gene transfer and the origins of prokaryotic groups. Annu. Rev. Genet. 37 283-328 (2003)

54. Darling, A. E., Miklos, I. \& Ragan, M. A. Dynamics of genome rearrangement in bacterial populations. PLoS Genet. 4, e1000128 (2008).

55. Kinsella, R. J., Fitzpatrick, D. A., Creevey, C. J. \& Mclnerney, J. O. Fatty acid biosynthesis in Mycobacterium tuberculosis: lateral gene transfer, adaptive evolution, and gene duplication. Proc. Nat Acad. Sci. USA 100, 10320-10325 (2003).

56. Jang, J., Becq, J., Gicquel, B., Deschavanne, P. \& Neyrolles, O. Horizontally acquired genomic islands in the tubercle bacilli. Trends Microbiol. 16, 303-308 (2008).

57. Becq, J. et al. Contribution of horizontally acquired genomic islands to the evolution of the tubercle bacilli. Mol. Biol. Evol. 24, 1861-1871 (2007).

58. Stinear, T. P. et al. Insights from the complete genome sequence of Mycobacterium marinum on the evolution of Mycobacterium tuberculosis. Genome Res. 18, 729-741 (2008)

59. Veyrier, F. J., Dufort, A. \& Behr, M. A. The rise and fall of the Mycobacterium tuberculosis genome. Trends Microbiol. 19, 156-161 (2011).

60. Supply, P. et al. Linkage disequilibrium between minisatellite loci supports clonal evolution of Mycobacterium tuberculosis in a high tuberculosis incidence area. Mol. Microbiol. 47, 529-538 (2003).

61. Hirsh, A. E., Tsolaki, A. G., DeRiemer, K. Feldman, M. W. \& Small, P. M. Stable association between strains of Mycobacterium tuberculosis and their human host populations. Proc. Natl Acad. Sci. USA 101, 4871-4876 (2004).

62. Namouchi, A., Didelot, X., Schock, U., Gicquel, B. \& Rocha, E. P. After the bottleneck: genome-wide diversification of the Mycobacterium tuberculosis complex by mutation, recombination, and natural selection. Genome Res. 22, 721-734 (2012).

63. Tsolaki, A. G. et al. Functional and evolutionary genomics of Mycobacterium tuberculosis: insights from genomic deletions in 100 strains. Proc. Natl Acad. Sci. USA 101, 4865-4870 (2004).

64. Ho, T. B., Robertson, B. D., Taylor, G. M., Shaw, R. J. $\delta$ Young, D. B. Comparison of Mycobacterium tuberculosis genomes reveals frequent deletions in a $20 \mathrm{~kb}$ variable region in clinical isolates. Yeast 17 272-282 (2000)

65. Sampson, S. L., Richardson, M., Van Helden, P. D. \& Warren, R. M. IS6110-mediated deletion polymorphism in isogenic strains of Mycobacterium tuberculosis. J. Clin. Microbiol. 42, 895-898 (2004)

66. Sampson, S. L. et al. IS6110-mediated deletion polymorphism in the direct repeat region of clinical isolates of Mycobacterium tuberculosis. J. Bacteriol. $185,2856-2866$ (2003).

67. Alexander, D. C., Turenne, C. Y. \& Behr, M. A Insertion and deletion events that define the pathogen Mycobacterium avium subsp. paratuberculosis. J. Bacteriol. 191, 1018-1025 (2009)

68. Domenech, P., Kolly, G. S., Leon-Solis, L., Fallow, A. \& Reed, M. B. Massive gene duplication event among clinical isolates of the Mycobacterium tuberculosis W Beijing family. J. Bacteriol. 192, 4562-4570 (2010).

69. Weiner, B. et al. Independent large scale duplications in multiple $M$. tuberculosis lineages overlapping the same genomic region. PLOS ONE 7, e26038 (2012). 
70. McDonough, M. A. \& Butterton, J. R. Spontaneous tandem amplification and deletion of the shiga toxin operon in Shigella dysenteriae 1. Mol. Microbiol. 34 1058-1069 (1999).

71. Bennett, P. M. Genome plasticity: insertion sequence elements, transposons and integrons, and DNA rearrangement. Methods Mol. Biol. 266, 71-113 (2004).

72. Ford, C. B. et al. Use of whole genome sequencing to estimate the mutation rate of Mycobacterium tuberculosis during latent infection. Nature Genet. $\mathbf{4 3}$, 482-486 (2011).

This WGS analysis of M. tuberculosis Erdman strains that have been recovered from infected cynomolgus macaques was used to estimate the mutation rate of $M$. tuberculosis in vivo. Surprisingly, the results suggest that the mutation rates are similar during active and latent disease.

73. Ford, C. B. et al. Mycobacterium tuberculosis mutation rate estimates from different lineages predict substantial differences in the emergence of drugresistant tuberculosis. Nature Genet. 45, 784-790 (2013).

This paper shows that $M$. tuberculosis strains from lineage 2 have a higher mutation rate and thus acquire drug resistance more rapidly than strains from lineage 4.

74. McGrath, M., Gey van Pittius, N. C., van Helden, P. D. Warren, R. M. \& Warner, D. F. Mutation rate and the emergence of drug resistance in Mycobacterium tuberculosis. J. Antimicrob. Chemother. 69, 292-302 (2013).

75. Gill, W. P. et al. A replication clock for Mycobacterium tuberculosis. Nature Med. 15, 211-214 (2009).

76. Munoz-Elias, E. J. et al. Replication dynamics of Mycobacterium tuberculosis in chronically infected mice. Infect. Immun. 73, 546-551 (2005)

77. Cohen, N. R., Lobritz, M. A. \& Collins, J. J. Microbial persistence and the road to drug resistance. Cell Host Microbe 13, 632-642 (2013).

78. Kohanski, M. A., DePristo, M. A. \& Collins, J. J. Sublethal antibiotic treatment leads to multidrug resistance via radical-induced mutagenesis. Mol. Cell 37, 311-320 (2010)

79. Dwyer, D. J., Kohanski, M. A. \& Collins, J. J. Role of reactive oxygen species in antibiotic action and resistance. Curr. Opin. Microbiol. 12, 482-489 (2009).

80. loerger, T. R. et al. Genome analysis of multiand extensively-drug-resistant tuberculosis from KwaZulu-Natal, South Africa. PLoS ONE 4, e7778 (2009).

81. loerger, T. R. et al. The non-clonality of drug resistance in Beijing-genotype isolates of Mycobacterium tuberculosis from the Western Cape of South Africa. BMC Genomics 11, 670 (2010).

82. Mariam, S. H., Werngren, J., Aronsson, J., Hoffner, S. $\Sigma$ Andersson, D. I. Dynamics of antibiotic resistant Mycobacterium tuberculosis during long-term infection and antibiotic treatment. PLOS ONE 6 , e21147 (2011)

83. Casali, N. et al. Microevolution of extensively drugresistant tuberculosis in Russia. Genome Res. 22, 735-745 (2012)

84. Sun G et al. Dynamic population changes in Mycobacterium tuberculosis during acquisition and fixation of drug resistance in patients. J. Infect. Dis. 206, 1724-1733 (2012)

This WGS study of the bacterial population from serial samples from three patients during the acquisition of drug resistance reveals that different resistance mutations can independently arise multiple times in an individual patient.

85. Fortune, S. M. The surprising diversity of Mycobacterium tuberculosis: change you can believe in. J. Infect. Dis. 206, 1642-1644 (2012).

86. Nachega, J. B. \& Chaisson, R. E. Tuberculosis drug resistance: a global threat. Clin. Infect. Dis. 36 S24-S30 (2003)

87. Cirz, R. T. \& Romesberg, F. E. Induction and inhibition of ciprofloxacin resistance-conferring mutations in hypermutator bacteria. Antimicrob. Agents Chemother. 50, 220-225 (2006)

88. Boshoff, H. I., Reed, M. B., Barry, C. E., 3rd \& Mizrahi, V. DnaE2 polymerase contributes to in vivo survival and the emergence of drug resistance in Mycobacterium tuberculosis. Cell 113, 183-193 (2003).

89. Drobniewski, F. et al. Drug-resistant tuberculosis, clinical virulence, and the dominance of the Beijing strain family in Russia. JAMA 293, 2726-2731 (2005).
90. Johnson, R. et al. Drug-resistant tuberculosis epidemic in the Western Cape driven by a virulent Beijing genotype strain. Int. J. Tuberc Lung Dis. 14, 119-121 (2010).

91. Cohen-Bacrie, S. et al. Imported extensively drugresistant Mycobacterium tuberculosis Beijing genotype, Marseilles, France, 2011. Euro Surveill 16 19846 (2011)

92. Kubica, T. et al. The Beijing genotype is a major cause of drug-resistant tuberculosis in Kazakhstan Int. J. Tuberc Lung Dis. 9, 646-653 (2005).

93. Mestre, O. et al. Phylogeny of Mycobacterium tuberculosis Beijing strains constructed from polymorphisms in genes involved in DNA replication, recombination and repair. PLOS ONE 6, e 16020 (2011)

94. Muller, B., Borrell, S., Rose, G. \& Gagneux, S. The heterogeneous evolution of multidrug-resistant Mycobacterium tuberculosis. Trends Genet. 29 , 160-169 (2013).

95. Gagneux, S. et al. The competitive cost of antibiotic resistance in Mycobacterium tuberculosis. Science 312, 1944-1946 (2006)

This paper shows that clinically derived drug-resistant strains most frequently harbour mutations with small or no fitness cost. This contrasts with laboratory-derived resistant strains, the mutations in which frequently affect competitive fitness.

96. Billington, O. J., McHugh, T. D. \& Gillespie, S. H Physiological cost of rifampin resistance induced in vitro in Mycobacterium tuberculosis. Antimicrob. Agents Chemother. 43, 1866-1869 (1999).

97. Davies, A. P. et al. Comparison of fitness of two isolates of Mycobacterium tuberculosis, one of which had developed multi-drug resistance during the course of treatment. J. Infect. 41, 184-187 (2000).

98. Pym, A. S., Saint-Joanis, B. \& Cole, S. T. Effect of katG mutations on the virulence of Mycobacterium tuberculosis and the implication for transmission in humans. Infect. Immun. 70, 4955-4960 (2002).

99. Sander, P. et al. Fitness cost of chromosomal drug resistance-conferring mutations. Antimicrob. Agents Chemother. 46, 1204-1211 (2002).

100. Bottger, E. C., Springer, B., Pletschette, M. \& Sander, P. Fitness of antibiotic-resistant microorganisms and compensatory mutations. Nature Med. 4, 1343-1344 (1998).

101. Comas, I. et al. Whole-genome sequencing of rifampicin-resistant Mycobacterium tuberculosis strains identifies compensatory mutations in RNA polymerase genes. Nature Genet. 44, 106-110 (2011).

102. de Vos, M. et al. Putative compensatory mutations in the $r p o C$ gene of rifampin-resistant Mycobacterium tuberculosis are associated with ongoing transmission. Antimicrob. Agents Chemother. 57, 827-832 (2013).

103. Galagan, J. E. et al. The Mycobacterium tuberculosis regulatory network and hypoxia. Nature 499 178-183 (2013).

This study presents a comprehensive mapping of the $M$. tuberculosis regulatory network, which suggests complex regulatory links between processes that are required for adaptations to the host environment. The results also provide the most complete map of transcription factor binding for any bacterium so far and indicate that transcription factor interactions are more diverse than previously thought.

104. Robertson, G. et al. Genome-wide profiles of STAT1 DNA association using chromatin immunoprecipitation and massively parallel sequencing. Nature Methods 4 651-657 (2007).

105. Mikkelsen, T. S. et al. Genome-wide maps of chromatin state in pluripotent and lineage-committed cells Nature 448, 553-560 (2007).

106. Johnson, D. S., Mortazavi, A., Myers, R. M. \& Wold, B. Genome-wide mapping of in vivo protein-DNA interactions. Science 316, 1497-1502 (2007).

107. Galagan, J., Lyubetskaya, A. \& Gomes, A. ChIP-seq and the complexity of bacterial transcriptional regulation. Curr. Top. Microbiol. Immunol. 363 43-68 (2013).

108. Jaini, S. et al. Molecular Genetics of Mycobacteria 2nd edn (eds Hatfull, G. \& Jacobs, W. R. Jr.) (ASM Press, in the press).

109. Farnham, P. J. Insights from genomic profiling of transcription factors. Nature Rev. Genet. 10, 605-616 (2009)

110. Ideker, T. \& Sharan, R. Protein networks in disease. Genome Res. 18, 644 (2013)

111. Alon, U. Network motifs: theory and experimental approaches. Nature Rev. Genet. 8, 450-461 (2007).
112. Guelzim, N., Bottani, S., Bourgine, P. \& Kepes, F. Topological and causal structure of the yeast transcriptional regulatory network. Nature Genet. 31 60-63 (2002).

113. Alon, U. An Introduction to Systems Biology: Design Principles of Biological Circuits (Chapman and Hall/ CRC, 2006).

114. Levine, J. H., Lin, Y. \& Elowitz, M. B. Functional roles of pulsing in genetic circuits. Science 342 , 1193-1200 (2013)

115. Bennett, M. R. \& Hasty, J. Microfluidic devices for measuring gene network dynamics in single cells. Nature Rev. Genet. 10, 628-638 (2009).

116. Yosef, N. \& Regev, A. Impulse control: temporal dynamics in gene transcription. Cell 144, 886-896 (2011)

117. Wakamoto, Y. et al. Dynamic persistence of antibiotic-stressed mycobacteria. Science 339, 91-95 (2013).

118. Singh, A. et al. Mycobacterium tuberculosis WhiB3 maintains redox homeostasis by regulating virulence lipid anabolism to modulate macrophage response. PLoS Pathog. 5, e1000545 (2009).

119. Schnappinger, D. et al. Transcriptional adaptation of Mycobacterium tuberculosis within macrophages: insights into the phagosomal environment. J. Exp. Med. 198, 693-704 (2003)

120. Rohde, K., Yates, R. M., Purdy, G. E. \& Russell, D. G Mycobacterium tuberculosis and the environment within the phagosome. Immunol. Rev. 219, 37-54 (2007).

121. Yang, X., Nesbitt, N. M., Dubnau, E., Smith, I. \& Sampson, N. S. Cholesterol metabolism increases the metabolic pool of propionate in Mycobacterium tuberculosis. Biochemistry 48, 3819-3821 (2009).

122. Kendall, S. L. et al. Cholesterol utilization in mycobacteria is controlled by two TetR-type transcriptional regulators: kstR and kstR2. Microbiology 156, 1362-1371 (2010).

123. Kendall, S. L. et al. A highly conserved transcriptional repressor controls a large regulon involved in lipid degradation in Mycobacterium smegmatis and Mycobacterium tuberculosis. Mol. Microbiol. 65 684-699 (2007)

124. Nesbitt, N. M. et al. A thiolase of Mycobacterium tuberculosis is required for virulence and production of androstenedione and androstadienedione from cholesterol. Infect. Immun. 78, 275-282 (2010).

125. Uhia, I., Galan, B., Medrano, F. J. \& Garcia, J. L. Characterization of the KstR-dependent promoter of the first step of cholesterol degradative pathway in Mycobacterium smegmatis. Microbiology 157 2670-2680 (2011)

126. Munoz-Elias, E. J. \& McKinney, J. D. Mycobacterium tuberculosis isocitrate lyases 1 and 2 are jointly required for in vivo growth and virulence. Nature Med. 11, 638-644 (2005)

127. Daniel, J., Maamar, H., Deb, C., Sirakova, T. D. \& Kolattukudy, P. E. Mycobacterium tuberculosis uses host triacylglycerol to accumulate lipid droplets and acquires a dormancy-like phenotype in lipid-loaded macrophages. PLoS Pathog. 7, e 1002093 (2011).

128. Low, K. L. et al. Triacylglycerol utilization is required for regrowth of in vitro hypoxic nonreplicating Mycobacterium bovis bacillus Calmette-Guerin. J. Bacteriol. 191, 5037-5043 (2009).

129. Peyron, P. et al. Foamy macrophages from tuberculous patients' granulomas constitute a nutrient-rich reservoir for $M$. tuberculosis persistence. PLoS Pathog. 4, e1000204 (2008).

This paper shows that oxygenated mycolic acids from $M$. tuberculosis are sufficient to induce the differentiation of macrophages into foamy macrophages, which contain lipid bodies that have been shown to be accessible to $M$. tuberculosis as a potential nutrient source.

130. Russell, D. G., Cardona, P. J., Kim, M. J., Allain, S. \& Altare, F. Foamy macrophages and the progression of the human tuberculosis granuloma. Nature Immunol. 10, 943-948 (2009).

131. Singh, A. et al. Mycobacterium tuberculosis WhiB3 responds to $\mathrm{O}_{2}$ and nitric oxide via its [4Fe-4S] cluster and is essential for nutrient starvation survival. Proc. Natl Acad. Sci. USA 104 11562-11567 (2007)

132. Russell, D. G. The evolutionary pressures that have molded Mycobacterium tuberculosis into an infectious adjuvant. Curr. Opin. Microbiol. 16, 78-84 (2013).

133. Russell, D. G. Mycobacterium tuberculosis and the intimate discourse of a chronic infection. Immunol. Rev. 240, 252-268 (2011). 
134. Russell, D. G., Mwandumba, H. C. \& Rhoades, E. E. Mycobacterium and the coat of many lipids. J. Cell Biol. 158, 421-426 (2002)

135. Russell, D. G. et al. Mycobacterium tuberculosis wears what it eats. Cell Host Microbe 8, 68-76 (2010).

136. Ehlers, S. \& Schaible, U. E. The granuloma in tuberculosis: dynamics of a host-pathogen collusion. Front. Immunol. 3, 411 (2012)

137. Comas, I. \& Gagneux, S. A role for systems epidemiology in tuberculosis research. Trends Microbiol. 19, 492-500 (2011)

138. Pawlowski, A., Jansson, M., Skold, M. Rottenberg, M. E. \& Kallenius, G. Tuberculosis and HIV co-infection. PLoS Pathog. 8, e1002464 (2012).

139. Dooley, K. E. \& Chaisson, R. E. Tuberculosis and diabetes mellitus: convergence of two epidemics. Lancet Infect. Dis. 9, 737-746 (2009).

140. Brothwell, D. R. \& Sandison, A. T. Diseases in Antiquity: a Survey of the Diseases, Injuries, and Surgery of Early Populations (C. C. Thomas, 1967).

141. Prasad, P. V. General medicine in Atharvaveda with special reference to Yaksma (consumption/ tuberculosis). Bull. Indian Inst. Hist. Med. Hyderabad 32, 1-14 (2002)

142. Daniel, V. S. \& Daniel, T. M. Old Testament biblical references to tuberculosis. Clin. Infect. Dis. 29 1557-1558 (1999).

143. Roberts, C. A. \& Buikstra, J. E. The Bioarchaeology of Tuberculosis: a Global View on a Reemerging Disease (Univ. Press of Florida, 2008).

144. Donoghue, H. D. et al. Tuberculosis: from prehistory to Robert Koch, as revealed by ancient DNA. Lancet Infect. Dis. 4, 584-592 (2004).

145. Zink, A. et al. Molecular history of tuberculosis from ancient mummies and skeletons. Int. J. Osteoarchaeol. 17, 380 (2007)

146. Bouwman, A. S. et al. Genotype of a historic strain of Mycobacterium tuberculosis. Proc. Natl Acad. Sci. 109, 18511-18516 (2012)

147. Zimmerman, M. R. Pulmonary and osseous tuberculosis in an Egyptian mummy. Bull. N. Y. Acad. Med. 55, 604-608 (1979).

148. Crubezy, E. et al. Identification of Mycobacterium DNA in an Egyptian Pott's disease of 5,400 years old C. R. Acad. Sci. III 321, 941-951 (1998).

149. Zink, A., Haas, C. J., Reischl, U., Szeimies, U. $\delta$ Nerlich, A. G. Molecular analysis of skeletal tuberculosis in an ancient Egyptian population. J. Med. Microbiol. 50, 355-366 (2001)

150. Zink, A. R., Grabner, W., Reischl, U., Wolf, H. \& Nerlich, A. G. Molecular study on human tuberculosis in three geographically distinct and time delineated populations from ancient Egypt. Epidemiol. Infect 130, 239-249 (2003).

151. Zink, A. R. et al. Characterization of Mycobacterium tuberculosis complex DNAs from Egyptian mummies by spoligotyping. J. Clin. Microbiol. 41, 359-367 (2003).

152. Nerlich, A. G. \& Losch, S. Paleopathology of human tuberculosis and the potential role of climate. Interdiscip Perspect. Infect. Dis. 2009, 437187 (2009).

153. Nicklisch, N. et al. Rib lesions in skeletons from early neolithic sites in Central Germany: on the trail of tuberculosis at the onset of agriculture. Am. J. Phys. Anthropol. 149, 391-404 (2012).

154. Formicola, V., Milanesi, Q. \& Scarsini, C. Evidence of spinal tuberculosis at the beginning of the fourth millennium вс from Arene Candide cave (Liguria, Italy). Am. J. Phys. Anthropol. 72, 1-6 (1987).
155. Sager, P., Schalimtzer, M. \& Moller-Christensen, V. A case of spondylitis tuberculosa in the Danish Neolithic Age. Dan Med. Bull. 19, 176-180 (1972).

156. Fusegawa, H. et al. Outbreak of tuberculosis in a 2000-year-old Chinese population. Kansenshogaku Zasshi 77, 146-149 (2003).

157. Hershkovitz, l. et al. Detection and molecular characterization of 9,000-year-old Mycobacterium tuberculosis from a Neolithic settlement in the Eastern Mediterranean. PLoS ONE 3, e3426 (2008).

158. Klaus, H. et al. Tuberculosis on the north coast of Peru: skeletal and molecular paleopathology of late pre-Hispanic and postcontact mycobacterial disease. J. Archaeol. Sci. 37, 2587 (2010).

159. Salo, W. L., Aufderheide, A. C., Buikstra, J. \& Holcomb, T. A. Identification of Mycobacterium tuberculosis DNA in a pre-Columbian Peruvian mummy. Proc. Natl Acad. Sci. USA 91, 2091-2094 (1994)

160. Arriaza, B. T. Salo, W. Aufderheide, A. C. \& Holcomb, T. A. Pre-Columbian tuberculosis in northern Chile: molecular and skeletal evidence. Am. J. Phys. Anthropol. 98, 37-45 (1995).

161. Rothschild, B. M. \& Martin, L. D. Did ice-age bovids spread tuberculosis? Naturwissenschaften 93 . 565-569 (2006).

162. Rothschild, B. M. \& Laub, R. Hyperdisease in the late Pleistocene: validation of an early 20th century hypothesis. Naturwissenschaften $93,557-564$ (2006).

163. Borgdorff, M. W. \& van Soolingen, D The re-emergence of tuberculosis: what have we learn from molecular epidemiology? Clin. Microbiol. Infect. 19, 889-901 (2013).

164. Gori, A. et al. Spoligotyping and Mycobacterium tuberculosis. Emerg. Infect. Dis. 11, 1242-1248 (2005).

165. Gori, A. et al. Comparison between spoligotyping and IS6110 restriction fragment length polymorphisms in molecular genotyping analysis of Mycobacterium tuberculosis strains. Mol. Cell Probes 19, 236-244 (2005).

166. Barrangou, R. et al. CRISPR provides acquired resistance against viruses in prokaryotes. Science 315, 1709-1712 (2007)

167. Marraffini, L. A. \& Sontheimer, E. J. CRISPR interference limits horizontal gene transfer in staphylococci by targeting DNA. Science 322 1843-1845 (2008)

168. Schurch, A. C. \& van Soolingen, D. DNA fingerprinting of Mycobacterium tuberculosis: from phage typing to whole-genome sequencing. Infect. Genet. Evol. 12 602-609 (2012)

169. McAdam, R. A et al. Characterization of a Mycobacterium tuberculosis insertion sequence belonging to the IS3 family. Mol. Microbiol. 4 , 1607-1613 (1990)

170. Coros, A., DeConno, E. \& Derbyshire, K. M IS6110, a Mycobacterium tuberculosis complex specific insertion sequence, is also present in the genome of Mycobacterium smegmatis, suggestive of lateral gene transfer among mycobacterial species. J. Bacteriol. 190, 3408-3410 (2008)

171. Supply, P. et al. Proposal for standardization of optimized mycobacterial interspersed repetitive unit-variable-number tandem repeat typing of Mycobacterium tuberculosis. J. Clin. Microbiol. 44, 4498-4510 (2006).
172. Djelouadji, Z Arnold C. Gharbia, S., Raoult, D. $\bar{\alpha}$ Drancourt, M. Multispacer sequence typing for Mycobacterium tuberculosis genotyping. PLOS ONE 3, e2433 (2008)

173. Maccallum, I. et al. ALLPATHS 2: small genomes assembled accurately and with high continuity from short paired reads. Genome Biol. 10, R103 (2009).

174. Butler, J. et al. ALLPATHS: de novo assembly of whole-genome shotgun microreads. Genome Res. 18, 810-820 (2008)

175. Zerbino, D. R. Using the Velvet de novo assembler for short-read sequencing technologies. Curr. Protoc. Bioinformatics 31, 11.5.1-11.5.12 (2010).

176. Denisov, G. et al. Consensus generation and variant detection by Celera Assembler. Bioinformatics $\mathbf{2 4}$, 1035-1040 (2008)

177. Zhang, T., Luo, Y., Chen, Y., Li, X. \& Yu, J. BIGrat: a repeat resolver for pyrosequencing-based re-sequencing with Newbler. BMC Res. Notes 5, 567 (2012).

178. Quail, M. A. et al. A tale of three next generation sequencing platforms: comparison of Ion Torrent, Pacific Biosciences and Illumina MiSeq sequencers. BMC Genomics 13, 341 (2012).

179. Bashir, A. et al. A hybrid approach for the automated finishing of bacterial genomes. Nature Biotech. 30 701-707 (2012).

180. Koren, S. et al. Reducing assembly complexity of microbial genomes with single-molecule sequencing Genome Biol. 14, R101 (2013).

181. Walker, T. M. et al. Whole-genome sequencing to delineate Mycobacterium tuberculosis outbreaks: a retrospective observational study. Lancet Infect. Dis. 13, 137-146 (2013)

182. Gardy, J. L. et al. Whole-genome sequencing and social-network analysis of a tuberculosis outbreak. N. Engl. J. Med. 364, 730-739 (2011).

183. Bryant, J. M. et al. Whole-genome sequencing to establish relapse or re-infection with Mycobacterium tuberculosis: a retrospective observational study. Lancet Respir. Med. 1, 786-792 (2013)

Acknowledgements

The author thanks E. Azizi, A. Earl and B. Birren for their input and assistance. This project has been funded in whole or in part with Federal funds from the US National Institute of Allergy and Infectious Diseases, US National Institutes of Health, Department of Health and Human Services, under Contract Number HHSN272200800059C, and a grant from the Massachusetts Life Sciences Foundation.

Competing interests statement

The author declares no competing interests.

\section{FURTHER INFORMATION}

Tuberculist: http://tuberculist.epfl.ch

Tuberculosis Database: http://tbdb.org

uberculosis Phylogeographic Diversity Sequencing Project:

http://genome.tbdb.org/annotation/genome/tbdb/

ReseqStrainInfo.htm

SUPPLEMENTARY INFORMATION

See online article: $\underline{\mathrm{S}}$ (table) $\mid \underline{\mathrm{S} 2}$ (figure)

ALL LINKS ARE ACTIVE IN THE ONLINE PDF 